MATHEMATICS OF COMPUTATION

Volume 69, Number 232, Pages 1559-1575

S 0025-5718(99)01171-0

Article electronically published on August 19, 1999

\title{
ANALYSIS OF THE FINITE PRECISION BI-CONJUGATE GRADIENT ALGORITHM FOR NONSYMMETRIC LINEAR SYSTEMS
}

\author{
CHARLES H. TONG AND QIANG YE
}

\begin{abstract}
In this paper we analyze the bi-conjugate gradient algorithm in finite precision arithmetic, and suggest reasons for its often observed robustness. By using a tridiagonal structure, which is preserved by the finite precision bi-conjugate gradient iteration, we are able to bound its residual norm by a minimum polynomial of a perturbed matrix (i.e. the residual norm of the exact GMRES applied to a perturbed matrix) multiplied by an amplification factor. This shows that occurrence of near-breakdowns or loss of biorthogonality does not necessarily deter convergence of the residuals provided that the amplification factor remains bounded. Numerical examples are given to gain insights into these bounds.
\end{abstract}

\section{INTRODUCTION}

Since its introduction by Lanczos [16] and later re-discovery by Fletcher [7] in its present form, the bi-conjugate gradient (BiCG) algorithm has evolved many variations (e.g. CGS, BiCGSTAB, QMR, CSBCG [22, 25, 8, 2]), each of which was specially designed to overcome some of its inherent difficulties (the need for adjoint matrix vector product, potential breakdowns, erratic convergence behavior, etc.). However, it has been observed by Bank and Chan [2] and Tong [23] that, in many cases, BiCG may still be competitive (in terms of convergence and convergence rates), especially when coupled with no or relatively poor preconditioners.

One major concern in using BiCG is two types of potential breakdown problems, which can cause numerical instability. In addition, in finite precision arithmetic the biorthogonality is lost, as is experienced by other Lanczos-type algorithms. As a result, the finite precision BiCG iteration can deviate significantly from the exact one. However, in many cases where such difficulties arise, BiCG exhibits exceptional numerical robustness in practice as far as the convergence of the residual norm is concerned [2, 23]. Specifically, very often, occurrence of near-breakdowns or loss of biorthogonality does not deter convergence of the residuals. On the other

Received by the editor October 6, 1998.

1991 Mathematics Subject Classification. Primary 65F10, 65N20.

Key words and phrases. Bi-conjugate gradient algorithm, error analysis, convergence analysis, nonsymmetric linear systems.

The first author's research was supported by Research Grant Council of Hong Kong.

The second author's research was supported by Natural Sciences and Engineering Research Council of Canada. Part of this work was completed while this author visited Stanford University during the summer of 1995. He would like to thank Professor Gene Golub for providing this opportunity and for his great hospitality. 
hand, it has been observed by Golub and Overton [9, 10] that the preconditioned conjugate gradient method with inexact preconditioner, which amounts to relatively large perturbations to the CG recurrence, could still converge. These phenomena suggest that the residual convergence property of $\mathrm{BiCG}$ (or $\mathrm{CG}$ ) may be relatively insensitive to perturbations in the recurrence, even though many other properties such as biorthogonality are sensitive to them. However, there is no theoretical result to explain such robustness. It is the purpose of the present paper to study the sometimes surprising convergence behavior of $\mathrm{BiCG}$ in finite precision arithmetic. We remark that our intention is not to suggest that BiCG will be robust in all cases, as there are plenty of divergent examples of BiCG, but rather to understand when and why BiCG iterations show more stability than expected.

In exact arithmetic, bounds on approximation errors (or residuals) of the BiCG iteration have been obtained by Bank and Chan 2, showing the fast decrease of the errors under certain conditions. Furthermore, it was also shown recently by Barth and Manteufel [3] that the BiCG residual indeed gives optimal approximation from Krylov subspaces, considered in a certain metric. Since proving these results 2 relies on the Galerkin condition (i.e., bi-orthogonality) of BiCG, which is usually lost in finite precision arithmetic, it is difficult to apply or generalize these analyses to the finite precision case. Furthermore, occurrence of near-breakdown could cause $\mathrm{BiCG}$ to generate completely different sequences for exact arithmetic and for a finite precision arithmetic. Nevertherless, we will show in this paper that the convergence property of residuals may still be preserved.

We shall prove a posteriori residual bounds similar to those in [2] for the exact case, using an approach that is based on a tridiagonal structure implicit in the algorithm. This approach was also used by Ye [27] to analyze convergence of the Lanczos algorithms for eigenvalue problems. An advantage of analyzing BiCG using its tridiagonal structure is that our results include the finite precision case and the near-breakdown case, and explain its sometimes observed convergence under quite general conditions.

Finite precision analyses of conjugate gradient-type and Lanczos-type algorithms have played an important role in understanding these algorithms. The pioneering work is due to C. Paige [19 20] and A. Greenbaum [12. Paige showed in [19, 20] that the loss of orthogonality comes with but does not prevent convergence of the Ritz values, i.e., useful results can still be obtained from the algorithm even when the iterates deviate significantly from what would have been produced in exact arithmetic. A generalization to the nonsymmetric case was given by Bai [1, and the near-breakdowns and loss of biorthogonality were discussed by Day [5]. Greenbaum established backward stability results in a generalized sense [12, showing that the iterative residuals produced by the finite precision conjugate gradient algorithm are equivalent to what would have been produced by applying the exact CG to a larger matrix. Some estimates on the larger matrix were given, which may vary from step to step. It would be interesting to see if Greenbaum's backward stability results can be generalized to BiCG; we are not aware of any such generalization. One analysis is given by Cullum and Greenbaum [4], which relates BiCG type methods to QMR. We note that a recent work by Greenbaum, Druskin and Knizhnerman [15] on the finite precision $\mathrm{CG}$ also uses the approach of bounding the residuals. Other recent works on the finite precision CG include [13, 14, 18, 25].

The paper is organized as follows. In section 2, we review the BiCG algorithm and discuss its theoretical properties. We then present our results in section [3, with 
a roundoff error analysis in section 3.1 then various approximation bounds on the BiCG residuals in sections 3.2 and [3.3. We present our numerical experiments in section 4 and concluding remarks in section 5 .

Notation. We shall use the standard notation in numerical analysis. In particular, for the roundoff error analysis, absolute values and inequalities of matrices are componentwise, i.e., $\left|\left(a_{i j}\right)\right|=\left(\left|a_{i j}\right|\right)$, and $\left(a_{i j}\right)<\left(b_{i j}\right)$ means $a_{i j}<b_{i j} . A \otimes B$ denotes the Kronecker product of $A$ and $B$, and $\lambda(A)$ denotes the spectrum of $A$. $A^{+}$denotes the Moore-Penrose generalized inverse of $A$. $I_{n}$ is the $n \times n$ identity matrix $\left[e_{1}, \ldots, e_{n}\right]=I_{n} . \mathcal{P}_{n}$ denotes the set of polynomials of degree not exceeding $n$.

\section{The BiCG ALgORITHM}

The BiCG algorithm for solving the linear system $A x=b$ is implemented as a pair of coupled two-term recurrences as follows.

Input initial approximation $x_{1}$;

Initialize $r_{1}=p_{1}=\tilde{r}_{1}=\tilde{p}_{1}=b-A x_{1}$;

$\rho_{1}=\tilde{r}_{1}^{T} r_{1}$

For $n=1,2, \cdots \cdots$

$\sigma_{n}=\tilde{p}_{n}^{T} A p_{n}$

$\alpha_{n}=\frac{\rho_{n}}{\sigma_{n}}$

$r_{n+1}=r_{n}-\alpha_{n} A p_{n}$

$x_{n+1}=x_{n}+\alpha_{n} p_{n}$

$\tilde{r}_{n+1}=\tilde{r}_{n}-\alpha_{n} A^{T} \tilde{p}_{n}$

$\rho_{n+1}=\tilde{r}_{n+1}^{T} r_{n+1}$

$\beta_{n+1}=\frac{\rho_{n+1}}{\rho_{n}}$

$p_{n+1}=r_{n+1}+\beta_{n+1} p_{n}$

end for

$\tilde{p}_{n+1}=\tilde{r}_{n+1}+\beta_{n+1} \tilde{p}_{n}$

The algorithm breaks down if either $\sigma_{n}=0$ (called pivotal breakdown) or $\rho_{n}=0$ (breakdown in the underlying Lanczos process). Instability may be expected when the iteration is close to breakdown (called near-breakdown). We shall assume in this paper that no exact breakdown occurs.

The sequence generated by the algorithm satisfies the following biorthogonality and biconjugacy conditions:

$$
\tilde{r}_{n}^{T} r_{m}=0, \quad \tilde{p}_{n}^{T} A p_{m}=0, \quad \text { for } m \neq n .
$$

$r_{n+1}$ is called the computed residual, and in exact arithmetic, it is equal to the true residual $b-A x_{n+1}$. The biorthogonality of the residuals implies that $r_{n+1}$ is orthogonal to the dual Krylov subspace

$$
K_{n}\left(A^{T}, r_{1}\right)=\operatorname{span}\left\{r_{1}, A^{T} r_{1}, \cdots,\left(A^{T}\right)^{n-1} r_{1}\right\} .
$$

This is also called the Galerkian condition.

Writing $R_{n}=\left[r_{1}, \cdots, r_{n}\right]$ and $P_{n}=\left[p_{1}, \cdots, p_{n}\right]$, we can derive from the recurrence the following matrix relations:

$$
A P_{n}=R_{n} L_{n} \Lambda_{N}^{-1}-\frac{1}{\alpha_{n}} r_{n+1} e_{n}^{T} \quad \text { and } \quad R_{n}=P_{n} U_{n},
$$


where $\Lambda_{n}=\operatorname{diag}\left[\alpha_{1}, \cdots, \alpha_{n}\right], e_{n}^{T}=[0, \ldots, 0,1]$ and

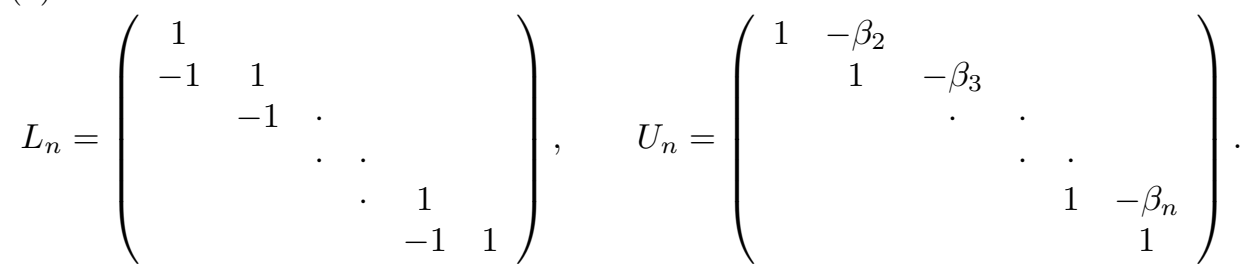

Combining the two equations in (1), we obtain the governing equation

$$
A R_{n}=R_{n} \hat{T}_{n}-\frac{1}{\alpha_{n}} r_{n+1} e_{n}^{T}
$$

where $\hat{T}_{n}=L_{n} \Lambda_{n}^{-1} U_{n}$ is an invertible tridiagonal matrix such that

$$
e_{n}^{T} \hat{T}_{n}^{-1} e_{1}=e_{n}^{T} U_{n}^{-1} \Lambda_{n} L_{n}^{-1} e_{1}=e_{n}^{T} \Lambda_{n} v=\alpha_{n},
$$

where $v=\left[\begin{array}{llll}1 & 1 & \cdots & 1\end{array}\right]^{T}$. Similar results hold for the dual sequences $\tilde{p}_{n}$ and $\tilde{r}_{n}$.

\section{Analysis of the finite precision BiCG}

In this section we present the main results in several subsections. We begin with an outline of the main ideas of this paper.

It is well known that the biorthogonality (or orthogonality in the symmetric case) in a Lanczos-type algorithm is usually lost in finite precision arithmetic, and thus the iterates computed in a finite precision arithmetic may differ significantly from the corresponding exact quantities. In addition to this difficulty, BiCG may encounter near-breakdowns, and as a result, large roundoff errors may occur at two possible places. One is in the computation of $\alpha$ 's and $\beta$ 's, and the other is in the computation of $r$ 's and $p$ 's. However, the errors in the computation of $\alpha$ 's and $\beta$ 's do not translate into errors in the governing equation (3), which depends on computations in the local vector recurrence only, not on how accurate the $\alpha$ 's and $\beta$ 's are. This is demonstrated in Sec. 3.1 by showing that (3) is still valid to within a small perturbation. So roundoff errors may cause the iterates to deviate from the exact ones and loss of biorthogonality; but they may not destroy the underlying tridiagonal structure in the BiCG algorithm. The importance of (3) was originally pointed out by Paige for the Lanczos algorithm and by Greenbaum for the CG algorithm.

We claim that the existence of this tridiagonal structure also plays a major role in the convergence of the BiCG residuals in a finite precision arithmetic. We shall prove in Sec. 3.2 some bounds on the computed residual $r_{n}$ based on a perturbed (3i) (see equation (11) below). The bounds are of a posteriori type and are in terms of the residual norm of the exact GMRES applied to a perturbed matrix multiplied by an amplification factor.

We mention that in finite precision arithmetic, the computed residuals $r_{n}$ differ from the true residual $b-A x_{n}$; however, they have the same magnitudes before roundoff error accumulation dominates (cf. [12, Theorem 2]). In investigating the robustness of BiCG, we are only interested in the convergence of $r_{n}$ because it is the convergence of $r_{n}$ that drives the convergence of the true residual $b-A x_{n}$. This is also consistent with the analysis of the CG case [12]. 
3.1. Roundoff error analysis of BiCG. We provide in this section a roundoff error analysis of the BiCG algorithm in finite precision arithmetic. A similar analysis for the Lanczos algorithm has been given in [1]. Our analysis is based on the following simplified model of roundoff errors in basic matrix computations [11, p. 66] (recall that inequalities are componentwise)

$$
\begin{gathered}
f l(\alpha x+y)=\alpha x+y+\mathbf{u} e \quad \text { with } \quad|e| \leq 2|\alpha x|+|y|+O(\mathbf{u}), \\
f l(A x)=A x+\mathbf{u} g \quad \text { with } \quad|g| \leq N|A||x|+O(\mathbf{u}),
\end{gathered}
$$

where $\mathbf{u}$ is the machine precision unit, $x, y \in R^{N}$, and $\alpha \in R$. Note that $O(\mathbf{u})$ denotes a term containing $\mathbf{u}$ and can be bounded rigorously.

For ease of notation, we shall use $r_{n}, x_{n}, p_{n}, \alpha_{n}, \beta_{n}$ etc. directly to denote the computed quantities in finite precision arithmetic in the rest of the paper.

Theorem 3.1. Let $\mathbf{u}$ be the machine precision unit and let $r_{n}, x_{n}, p_{n}, \alpha_{n}, \beta_{n}$ be the computed quantities in the finite precision BiCG algorithm. Then

$$
A R_{n}=R_{n} \hat{T}_{n}-\frac{1}{\alpha_{n}} r_{n+1} e_{n}^{T}+\mathbf{u} \hat{\Delta}_{n}
$$

where $R_{n}=\left[r_{1}, \cdots, r_{n}\right], \hat{T}_{n}=L_{n} \Lambda_{n}^{-1} U_{n}$ (given in 国) and $\hat{\Delta}_{n}=\left[\delta_{1}, \cdots, \delta_{n}\right]$ with

$$
\left|\delta_{i}\right| \leq\left((N+6)|A|+\frac{1}{\left|\alpha_{i}\right|}+\frac{\left|\beta_{i}\right|}{\left|\alpha_{i-1}\right|}\right)\left|r_{i}\right|+(2 N+7)|A|\left|p_{i}\right|+O(\mathbf{u}) .
$$

Proof. At the $n$th iteration, to compute $r_{n+1}$, we first compute $A p_{n}$ and have $f l\left(A p_{n}\right)=A p_{n}+g$ with $|g| \leq \mathbf{u} N|A|\left|p_{n}\right|+O\left(\mathbf{u}^{2}\right)$ by (5). Then

$$
\begin{aligned}
r_{n+1} & =f l\left(r_{n}-\alpha_{n} f l\left(A p_{n}\right)\right)=r_{n}-\alpha_{n} f l\left(A p_{n}\right)+g^{\prime} \\
& =r_{n}-\alpha_{n} A p_{n}+\alpha_{n} g+g^{\prime},
\end{aligned}
$$

where, by (4),

$$
\left|g^{\prime}\right| \leq \mathbf{u}\left(\left|r_{n}\right|+2\left|\alpha_{n}\right|\left|f l\left(A p_{n}\right)\right|+O(\mathbf{u})\right)
$$

Letting $\delta_{r_{n}}=\left(\alpha_{n} g+g^{\prime}\right) /\left(\mathbf{u}\left|\alpha_{n}\right|\right)$, we obtain

$$
\frac{1}{\alpha_{n}}\left(r_{n+1}-r_{n}\right)=-A p_{n}+\mathbf{u} \delta_{r_{n}}
$$

with $\left|\delta_{r_{n}}\right| \leq N|A|\left|p_{n}\right|+\left|r_{n}\right| /\left|\alpha_{n}\right|+2\left|A p_{n}\right|+O(\mathbf{u}) \leq\left|r_{n}\right| /\left|\alpha_{n}\right|+(N+2)|A|\left|p_{n}\right|+O(\mathbf{u})$. Similarly

$$
p_{n+1}=r_{n+1}+\beta_{n+1} p_{n}+\mathbf{u} \delta_{p_{n+1}}
$$

with $\left|\delta_{p_{n+1}}\right| \leq\left|r_{n+1}\right|+2\left|\beta_{n+1}\right|\left|p_{n}\right|+O(\mathbf{u})$. Writing $\Delta_{R_{n}}=\left[\delta_{r_{1}}, \cdots, \delta_{r_{n}}\right]$ and $\Delta_{P_{n}}=\left[0, \delta_{p_{2}}, \cdots, \delta_{p_{n}}\right]$, we obtain from the above

$$
A P_{n}=R_{n} L_{n} \Lambda_{N}^{-1}-\frac{1}{\alpha_{n}} r_{n+1} e_{n}^{T}+\mathbf{u} \Delta_{R_{n}} \quad \text { and } \quad R_{n}=P_{n} U_{n}+\mathbf{u} \Delta_{P_{n}}
$$

where $R_{n}$, etc., are defined in the same way as in section 2. Combining the two equations, we obtain

$$
A R_{n}=R_{n} \hat{T}_{n}-\frac{1}{\alpha_{n}} r_{n+1} e_{n}^{T}+\mathbf{u} \hat{\Delta}_{n}
$$


with $\hat{\Delta}_{n}=A \Delta_{P_{n}}+\Delta_{R_{n}} U_{n}$. Write $\hat{\Delta}_{n}=\left[\delta_{1}, \cdots, \delta_{n}\right]$. Then we have $\delta_{i}=A \delta_{p_{i}}+$ $\delta_{r_{i}}-\delta_{r_{i-1}} \beta_{i}$. So for $i \geq 2$, we have (with $N^{\prime}=N+2$ )

$$
\begin{aligned}
\left|\delta_{i}\right| \leq & |A|\left|r_{i}\right|+2|A|\left|\beta_{i}\right|\left|p_{i-1}\right|+\frac{\left|r_{i}\right|}{\left|\alpha_{i}\right|}+N^{\prime}|A|\left|p_{i}\right| \\
& +\left|\beta_{i}\right|\left(\frac{\left|r_{i-1}\right|}{\left|\alpha_{i-1}\right|}+N^{\prime}|A|\left|p_{i-1}\right|\right)+O(\mathbf{u}) \\
\leq & \left(|A|+\frac{1}{\left|\alpha_{i}\right|}+\frac{\left|\beta_{i}\right|}{\left|\alpha_{i-1}\right|}\right)\left|r_{i}\right|+N^{\prime}|A|\left|p_{i}\right|+\left(N^{\prime}+3\right)|A|\left(\left|p_{i}\right|+\left|r_{i}\right|\right)+O(\mathbf{u}) \\
\leq & \left(\left(N^{\prime}+4\right)|A|+\frac{1}{\left|\alpha_{i}\right|}+\frac{\left|\beta_{i}\right|}{\left|\alpha_{i-1}\right|}\right)\left|r_{i}\right|+\left(2 N^{\prime}+3\right)|A|\left|p_{i}\right|+O(\mathbf{u}),
\end{aligned}
$$

where we have used $\left|r_{i-1}\right| \leq\left|r_{i}\right|+\left|\alpha_{i-1}\right|\left|A p_{i-1}\right|+O(\mathbf{u})$ and $\left|\beta_{i} p_{i-1}\right| \leq\left|p_{i}\right|+$ $\left|r_{i}\right|+O(\mathbf{u})$. The same bound holds for the case $i=1$, and hence the theorem is proved.

Remark. We can conclude from bound (7) that the accuracy in the computations of the coefficients $\alpha$ and $\beta$ does not directly affect the perturbation $\mathbf{u} \hat{\Delta}_{n}$. In particular, occurrence of near-breakdowns may cause the computed $\alpha$ or $\beta$ and hence the iterates to be inaccurate, but it does not necessarily cause a large error $\delta_{i}$ (see Example 2 in Sec. 4), and thus the fundamental equation (6) is still nearly satisfied. Also note that $\left|p_{i}\right| \leq\left|A^{-1}\right|\left(\left|r_{i}\right|+\left|r_{i+1}\right|\right) /\left|\alpha_{i}\right|+O(\mathbf{u})$, and therefore the relative magnitude of the perturbation $\delta_{i} /\left\|r_{i}\right\|$ does depend on the magnitude of $1 / \alpha_{i}$ and $\beta_{i}$.

In our later analysis, it is more convenient to work with an equivalent form of (6) with $r_{i}$ scaled to the unit norm. It will also become clear that only the relative perturbation $\delta_{i} /\left\|r_{i}\right\|$ is of importance. Let

$$
D_{n}=\operatorname{diag}\left\{\left\|r_{1}\right\|, \cdots,\left\|r_{n}\right\|\right\} \text { and } Z_{n}=\left[z_{1}, \cdots, z_{n}\right]=R_{n} D_{n}^{-1} .
$$

Then we obtain scaled (6) with $\left\|z_{i}\right\|=1$ as

$$
A Z_{n}=Z_{n} T_{n}-\frac{1}{\alpha_{n}^{\prime}} \frac{r_{n+1}}{\left\|r_{1}\right\|} e_{n}^{T}+\mathbf{u} \Delta_{n}
$$

where $T_{n}=D_{n} \hat{T}_{n} D_{n}^{-1}$ is an invertible tridiagonal matrix, $\alpha_{n}^{\prime}=\left\|r_{n}\right\| \alpha_{n} /\left\|r_{1}\right\|=$ $e_{n}^{T} T_{n}^{-1} e_{1}$ and

$$
\Delta_{n}=\hat{\Delta}_{n} D_{n}^{-1}=\left[\delta_{1} /\left\|r_{1}\right\|, \cdots, \delta_{n} /\left\|r_{n}\right\|\right] .
$$

This will be the only equation that we assume for BiCG in the rest of this section.

3.2. Bounds on $\left\|r_{n+1}\right\|$ for the finite precision BiCG. In this section, we present the main result on bounding the computed residual (Theorem 3.6). We first give a few lemmas. Recall that $\mathcal{P}_{n}$ denote the set of polynomials of degree not exceeding $n$.

Lemma 3.2. Assume $A Z_{n}=Z_{n} T_{n}-\frac{1}{\alpha_{n}^{\prime}} \frac{r_{n+1}}{\left\|r_{1}\right\|} e_{n}^{T}$ with $e_{n}^{T} T_{n}^{-1} e_{1}=\alpha_{n}^{\prime}$ and $r_{1}=$ $\left\|r_{1}\right\| z_{1}$. Then, for any polynomial $p(x)=\sum_{k=0}^{n} \psi_{k} x^{k}$ of degree not exceeding $n$,

$$
p(A) z_{1}=Z_{n} p\left(T_{n}\right) e_{1}+c_{n} r_{n+1},
$$

where $c_{n}=-\psi_{n}\left(\alpha_{1} \cdots \alpha_{n}\left\|r_{1}\right\|\right)^{-1}$. 
Proof. We first prove by induction that for any $k$ with $1 \leq k \leq n-1$,

$$
A^{k} Z_{n} e_{1}=Z_{n} T_{n}^{k} e_{1} \text {. }
$$

Clearly it is true for $k=1$. Assume that (13) is true for some $k \leq n-2$. First, we have $e_{n}^{T} T_{n}^{k} e_{1}=0$ (see [27, Lemma 3.1]). Then

$$
\begin{aligned}
A^{k+1} Z_{n} e_{1} & =A Z_{n} T_{n}^{k} e_{1}=\left(Z_{n} T_{n}-\frac{1}{\alpha_{n}^{\prime}\left\|r_{1}\right\|} r_{n+1} e_{n}^{T}\right) T_{n}^{k} e_{1} \\
& =Z_{n} T_{n}^{k+1} e_{1} .
\end{aligned}
$$

Therefore (13) holds. Consider now

$$
\begin{aligned}
A^{n} Z_{n} e_{1} & =A A^{n-1} Z_{n} e_{1}=A Z_{n} T_{n}^{n-1} e_{1} \\
& =\left(Z_{n} T_{n}-\frac{1}{\alpha_{n}^{\prime}\left\|r_{1}\right\|} r_{n+1} e_{n}^{T}\right) T_{n}^{n-1} e_{1} \\
& =Z_{n} T_{n}^{n} e_{1}-\frac{1}{\alpha_{1} \cdots \alpha_{n}\left\|r_{1}\right\|} r_{n+1},
\end{aligned}
$$

where $e_{n}^{T} T_{n}^{n-1} e_{1}=\left\|r_{n}\right\|\left(\alpha_{1} \cdots \alpha_{n-1}\left\|r_{1}\right\|\right)^{-1}$ (see [27, Theorem 3.2]). Combining the above with (13), we obtain (12).

Note that the above lemma also holds if $T_{n}$ is Hessenberg. In particular, if $p$ is a polynomial of degree $n-1$ (i.e. $\psi_{n}=0$ ), then (12) becomes $p(A) z_{1}=Z_{n} p\left(T_{n}\right) e_{1}$, a case that has been proved in 1721 .

From this lemma, we have the following identity concerning $r_{n+1}$.

\section{Lemma 3.3. Assume}

$$
A Z_{n}=Z_{n} T_{n}-\frac{1}{\alpha_{n}^{\prime}} \frac{r_{n+1}}{\left\|r_{1}\right\|} e_{n}^{T}
$$

with $e_{n}^{T} T_{n}^{-1} e_{1}=\alpha_{n}^{\prime}$ and $r_{1}=\left\|r_{1}\right\| z_{1}$, and assume that $V^{T} \in R^{n \times N}$ is a matrix such that $V^{T} Z_{n}=I$ and $V^{T} r_{n+1}=0$. Then for any polynomial $p(x)$ of degree not exceeding $n$ with $p(0)=1$, we have

$$
r_{n+1}=\left(I-A Z_{n} T_{n}^{-1} V^{T}\right) p(A) r_{1} .
$$

Proof. First, multiplying (14) by $T_{n}^{-1} e_{1}$, we obtain

$$
r_{n+1} /\left\|r_{1}\right\|=z_{1}-A Z_{n} T_{n}^{-1} e_{1} .
$$

Write $p(x)=1+x q(x)$, with $q(x)=\sum_{k=0}^{n-1} \psi_{k+1} x^{k}$ a polynomial of degree not exceeding $n-1$. Then

$$
\begin{aligned}
r_{n+1} /\left\|r_{1}\right\| & =z_{1}-A Z_{n} T_{n}^{-1} e_{1}-p(A) z_{1}+p(A) z_{1} \\
& =-A q(A) z_{1}-A Z_{n} T_{n}^{-1} e_{1}+p(A) z_{1} \\
& =-A Z_{n} q\left(T_{n}\right) e_{1}-A Z_{n} T_{n}^{-1} e_{1}+p(A) z_{1} \\
& =-A Z_{n}\left(q\left(T_{n}\right)+T_{n}^{-1}\right) e_{1}+p(A) z_{1} \\
& =-A Z_{n} T_{n}^{-1} p\left(T_{n}\right) e_{1}+p(A) z_{1},
\end{aligned}
$$

where we note that by Lemma 3.2, $A q(A) z_{1}=A q(A) Z_{n} e_{1}=A Z_{n} q\left(T_{n}\right) e_{1}$ with $\operatorname{deg}(q)<n$.

Now, for the polynomial $p$, equation (12) holds. Multiplying it by $V^{T}$, we obtain

$$
V^{T} p(A) z_{1}=p\left(T_{n}\right) e_{1}
$$


Finally, substituting $p\left(T_{n}\right) e_{1}$ into the equation above, we have

$$
\frac{r_{n+1}}{\left\|r_{1}\right\|}=\left(I-A Z_{n} T_{n}^{-1} V^{T}\right) p(A) z_{1}
$$

Now, the lemma follows from using $r_{1}=\left\|r_{1}\right\| z_{1}$.

Note that the matrix $V$ above exists if and only if $z_{1}, \cdots, z_{n}, z_{n+1}$ are linearly independent. In the case of exact BiCG, this is the case because of biorthogonality. Indeed, a natural choice for $V$ is $V^{T}=\left(\hat{R}_{n}^{T} R_{n}\right)^{-1} \hat{R}_{n}^{T}$, since by the biorthogonality condition $\hat{R}_{n}^{T} R_{n}=D$ is a diagonal matrix, and $\hat{R}_{n}^{T} r_{n+1}=0$. Using this $V$, the lemma leads to the known results for the exact BiCG [2].

When an biorthogonal basis is not explicitly available, the next lemma gives a construction of $V$.

Lemma 3.4. Assume that $z_{1}, z_{2}, \cdots, z_{n}, z_{n+1} \in R^{N}$ are linearly independent, and write $Z_{k}=\left[\begin{array}{llll}z_{1} & z_{2} & \cdots & z_{k}\end{array}\right]$. Then $V_{0}^{T}=\left[\begin{array}{ll}I_{n} & 0\end{array}\right] Z_{n+1}^{+}$(i.e. the matrix consisting of the first $n$ rows of $Z_{n+1}^{+}$) has the property

$$
V_{0}^{T} Z_{n}=I \text { and } V_{0}^{T} z_{n+1}=0 .
$$

Furthermore, its spectral norm is minimal among all the matrices having this property.

Proof. From the definition of $V_{0}, Z_{n+1}^{+}=\left[V_{0}, v\right]^{T}$ for some $v$. Since $z_{1}, \cdots, z_{n}, z_{n+1}$ are linearly independent, $\left[V_{0}, v\right]^{T}\left[\begin{array}{ll}Z_{n} & z_{n+1}\end{array}\right]=Z_{n+1}^{+} Z_{n+1}=I$. Then $V_{0}^{T} Z_{n}=I_{n}$ and $V_{0}^{T} z_{n+1}=0$.

Now, if $V$ is another matrix having property (19), then $V^{T}\left[\begin{array}{ll}Z_{n} & z_{n+1}\end{array}\right]=\left[\begin{array}{ll}I & 0\end{array}\right]$. Thus $V^{T} Z_{n+1} Z_{n+1}^{+}=\left[\begin{array}{ll}I & 0\end{array}\right] Z_{n+1}^{+}=V_{0}^{T}$. Hence $\left\|V_{0}\right\| \leq\|V\| \cdot\left\|Z_{n+1} Z_{n+1}^{+}\right\| \leq$ $\|V\|$.

We now present our main result on bounding the residuals.

Theorem 3.5. Assume equation [11) and let $V_{0}^{T}=\left[\begin{array}{ll}I_{n} & 0\end{array}\right] Z_{n+1}^{+} \in \mathbf{R}^{n \times N}$ (the matrix consisting of the first $n$ rows of $Z_{n+1}^{+}$). If $z_{1}, z_{2}, \cdots, z_{n}, z_{n+1}$ are linearly independent, then

$$
\left\|r_{n+1}\right\| \leq\left(1+K_{n}\right) \min _{p \in \mathcal{P}_{n}, p(0)=1}\left\|p\left(A+\delta A_{n}\right) r_{1}\right\|,
$$

where $K_{n}=\left\|\left(A Z_{n}-\mathbf{u} \Delta_{n}\right) T_{n}^{-1} V_{0}^{T}\right\|$ and $\delta A_{n}=-\mathbf{u} \Delta_{n} Z_{n}^{+}$.

Furthermore, as $n$ increases, $\epsilon_{n}=\min _{p \in \mathcal{P}_{n}, p(0)=1}\left\|p\left(A+\delta A_{n}\right) r_{1}\right\|$ decreases monotonically.

Proof. Since $z_{1}, \cdots, z_{n}, z_{n+1}$ are linearly independent, $Z_{n}^{+} Z_{n}=I$. Then $\delta A_{n}=$ $-\mathbf{u} \Delta_{n} Z_{n}^{+} \in R^{N \times N}$ satisfies $\delta A_{n} Z_{n}=-\mathbf{u} \Delta_{n}$. Thus (11) can be rewritten as

$$
\left(A+\delta A_{n}\right) Z_{n}=Z_{n} T_{n}-\frac{1}{\alpha_{n}^{\prime}} \frac{r_{n+1}}{\left\|r_{1}\right\|} e_{n}^{T} .
$$

Now for any $p \in \mathcal{P}_{n}$ with $p(0)=1$, we use Lemmas 3.3 and 3.4 to obtain

$$
\begin{aligned}
r_{n+1} & =\left(I-\left(A+\delta A_{n}\right) Z_{n} T_{n}^{-1} V_{0}^{T}\right) \cdot p\left(A+\delta A_{n}\right) r_{1} \\
& =\left(I-\left(A Z_{n}-\mathbf{u} \Delta_{n}\right) T_{n}^{-1} V_{0}^{T}\right) \cdot p\left(A+\delta A_{n}\right) r_{1} .
\end{aligned}
$$

Thus

$$
\left\|r_{n+1}\right\| \leq\left(1+\left\|\left(A Z_{n}-\mathbf{u} \Delta_{n}\right) T_{n}^{-1} V_{0}^{T}\right\|\right)\left\|p\left(A+\delta A_{n}\right) r_{1}\right\| .
$$


Since this is true for any $p(x)$ with $p(0)=1$, the inequality is true for the minimizing polynomial, which leads to the bound.

For the second part, we show that for any polynomial $p$ with $\operatorname{deg}(p)=k<m$,

$$
p\left(A+\delta A_{m}\right) z_{1}=p\left(A+\delta A_{k}\right) z_{1},
$$

which leads to the monotonicity result

$$
\min _{p \in \mathcal{P}_{m}, p(0)=1}\left\|p\left(A+\delta A_{m}\right) z_{1}\right\| \leq \min _{p \in \mathcal{P}_{k}, p(0)=1}\left\|p\left(A+\delta A_{k}\right) z_{1}\right\| .
$$

For any $i$ with $0 \leq i \leq k-1, T_{m}^{i} e_{1}=\left(\begin{array}{c}T_{k}^{i} e_{1} \\ 0\end{array}\right)$ (see [27, Lemma 3.1]). Then using Lemma 3.2. $\left(A+\delta A_{m}\right)^{i} z_{1}=Z_{m} T_{m}^{i} e_{1}=Z_{k} T_{k}^{i} e_{1}=\left(A+\delta A_{k}\right)^{i} z_{1}$. Furthermore,

$$
\begin{aligned}
\left(A+\delta A_{m}\right)^{k} z_{1} & =\left(A+\delta A_{m}\right) Z_{m} T_{m}^{k-1} e_{1} \\
& =\left(Z_{m} T_{m}-\frac{1}{\alpha_{m}^{\prime}\left\|r_{1}\right\|} r_{m+1} e_{m}^{T}\right) T_{m}^{k-1} e_{1} \\
& =Z_{m} T_{m}\left(\begin{array}{c}
T_{k}^{k-1} e_{1} \\
0
\end{array}\right) \\
& =Z_{k} T_{k}^{k} e_{1}-\frac{\left\|r_{k+1}\right\|}{\alpha_{k}\left\|r_{k}\right\|} z_{k+1} e_{k}^{T} T_{k}^{k-1} e_{1} \\
& =\left(Z_{k} T_{k}-\frac{1}{\alpha_{k}^{\prime}\left\|r_{1}\right\|} r_{k+1} e_{k}^{T}\right) T_{k}^{k-1} e_{1} \\
& =\left(A+\delta A_{k}\right) Z_{k} T_{k}^{k-1} e_{1} \\
& =\left(A+\delta A_{k}\right)^{k} z_{1} .
\end{aligned}
$$

Thus, $p\left(A+\delta A_{m}\right) z_{1}=p\left(A+\delta A_{k}\right) z_{1}$, and the proof is complete.

Remark. For each $n, \epsilon_{n}=\min _{p \in \mathcal{P}_{n}, p(0)=1}\left\|p\left(A+\delta A_{n}\right) r_{1}\right\|$ is the $n$th residual norm of exact GMRES applied to the perturbed matrix $A+\delta A_{n}$. Explicit bounds on $\epsilon_{n}$ have been discussed extensively in the literature; see 24 for example. Our numerical experiments show that the perturbation term $\delta A_{n}$ has little effect, i.e. usually $\epsilon_{n} \sim \min _{p \in \mathcal{P}_{n}, p(0)=1}\left\|p(A) r_{1}\right\|$.

Remark. If $T_{n}$ is not too close to being singular, and the elements of the basis $z_{1}, \cdots, z_{n}, z_{n+1}$ are not too close to being linearly dependent, i.e. $\left\|T_{n}^{-1}\right\|$ and $\left\|V_{0}\right\| \leq$ $\left\|Z_{n+1}^{+}\right\|$are bounded, then it follows from

$$
K_{n} \leq\left(\sqrt{n}\|A\|+\mathbf{u}\left\|\Delta_{n}\right\|\right)\left\|T_{n}^{-1}\right\| \cdot\left\|V_{0}\right\|
$$

that the residual norm $\left\|r_{n+1}\right\|$ is within a moderate factor of $\epsilon_{n}$. Hence, convergence of the BiCG residual can be achieved provided $K_{n}$ increases at a rate slower than the rate at which $\epsilon_{n}$ decreases (see the examples in Sec. 4). In particular, this is still possible even when $\left\|\Delta_{n}\right\|$ is large (Example 1 in Sec. 4). We note that $K_{n}$ could grow out of bound; but this simply reflects the situations when BiCG diverges. Unfortunately $K_{n}$ cannot be determined a priori.

Remark. If $T_{n}$ is close to being singular at some step $n$, then $r_{n+1}$ becomes (relatively) large in that step; but if at the next step $T_{n+1}$ becomes well conditioned, $r_{n+2}$ will be small. So occurrence of a nearly singular $T_{n}$ would cause local spikes in the convergence curve but may not affect the global convergence trend even in finite precision arithmetic, assuming that not all $T_{n}$ are nearly singular. 
Remark. The above bound assumes linear independence among the $r_{n}$, and $\left\|V_{0}\right\|$ is a measure of the linear independence. It suggests the possible effect of the linear dependence on the convergence, which is known, for example, for the exact QMR (see [8]). However, this dependence on $\left\|V_{0}\right\|$ is not essential, and in the next subsection we give some results that avoid such an assumption at the expense of a more complicated bound.

3.3. Bounds for the linearly dependent case. While it is well-known that loss of linear independence among the $r_{n}$ could cause deterioration of convergence in $\mathrm{BiCG}$, there are cases where the convergence has been observed even when the linear independence of the $r_{n}$ is completely lost (the case $n>N$ being one such example). We next present two bounds that avoid the linear independence condition and may, therefore, partially explain the convergence in such situations.

First, recall that for $A \in \mathbf{R}^{N \times N}$ and $B \in \mathbf{R}^{n \times n}$, the matrix equation $A E-$ $E B=Z$ corresponds to a linear system with $A \otimes I_{n}-I_{N} \otimes B$ as the coefficient matrix. It has a unique solution if and only if $\lambda(A) \cap \lambda(B)=\emptyset$ or $\operatorname{sep}(A, B)=$ $\left\|\left(A \otimes I_{n}-I_{N} \otimes B\right)^{-1}\right\|^{-1}>0$ [11, p. 389]. Note that $\operatorname{sep}(A, B)$ depends on the spectral gap of $A$ and $B$.

Theorem 3.6. Assume equation (11) and let $\mu$ be a complex number such that $\operatorname{sep}\left(A-\mu I, T_{n}\right)>>0$. Then

$$
\left\|r_{n+1}\right\| \leq K_{n} \min _{p \in \mathcal{P}_{n}, p(0)=1}\left(\left\|p\left(T_{n}\right)\right\|+\|p(A-\mu I)\|\right)\left\|r_{1}\right\|,
$$

where

$$
K_{n}=\frac{\left(|\mu|+\operatorname{sep}\left(A-\mu I, T_{n}\right)\right) \sqrt{n}+\mathbf{u}\left\|\Delta_{n}\right\|_{F}}{\operatorname{sep}\left(A-\mu I, T_{n}\right)} \max \left\{1, \quad\|A-\mu I\| \cdot\left\|T_{n}^{-1}\right\|\right\}
$$

Proof. First, we rewrite (11) as

$$
(A-\mu I) Z_{n}=Z_{n} T_{n}-\frac{1}{\alpha_{n}^{\prime}\left\|r_{1}\right\|} r_{n+1} e_{n}^{T}+\mathbf{u} \Delta_{n}-\mu Z_{n} .
$$

Since $\operatorname{sep}\left(A-\mu I, T_{n}\right)>0$, the matrix equation

$$
(A-\mu I) E_{n}=E_{n} T_{n}-\mathbf{u} \Delta_{n}+\mu Z_{n}
$$

has a unique solution $E_{n}$ [11, p.389] with

$$
\left\|E_{n}\right\|_{F} \leq \frac{\left\|-\mathbf{u} \Delta_{n}+\mu Z_{n}\right\|_{F}}{\operatorname{sep}\left(A-\mu I, T_{n}\right)} \leq \frac{\mathbf{u}\left\|\Delta_{n}\right\|_{F}+|\mu| \sqrt{n}}{\operatorname{sep}\left(A-\mu I, T_{n}\right)} .
$$

Then

$$
(A-\mu I)\left(Z_{n}+E_{n}\right)=\left(Z_{n}+E_{n}\right) T_{n}-\frac{1}{\alpha_{n}^{\prime}} \frac{r_{n+1}}{\left\|r_{1}\right\|} e_{n}^{T} .
$$

Thus, for any $p \in \mathcal{P}_{n}$ with $p(0)=1$, we have by (17)

$$
\frac{r_{n+1}}{\left\|r_{1}\right\|}=p(A-\mu I)\left(Z_{n}+E_{n}\right) e_{1}-(A-\mu I)\left(Z_{n}+E_{n}\right) T_{n}^{-1} p\left(T_{n}\right) e_{1},
$$

and hence

$$
\begin{aligned}
\frac{\left\|r_{n+1}\right\|}{\left\|r_{1}\right\|} \leq & \left(\left\|Z_{n}\right\|+\left\|E_{n}\right\|\right)\|p(A-\mu I)\| \\
& +\|A-\mu I\|\left(\left\|Z_{n}\right\|+\left\|E_{n}\right\|\right)\left\|T_{n}^{-1}\right\|\left\|p\left(T_{n}\right) e_{1}\right\|,
\end{aligned}
$$

which leads to the theorem, since $\left\|Z_{n}\right\|+\left\|E_{n}\right\| \leq \sqrt{n}+\left\|E_{n}\right\|_{F}$. 
Again the bound contains two parts. $K_{n}$ depends mainly on $\left\|T_{n}^{-1}\right\|$ if the parameter $\mu$ has been chosen to create a large $\operatorname{sep}\left(A-\mu I, T_{n}\right)$. The second part now depends on minimization of $p\left(T_{n}\right)$ in addition to $p(A-\mu I)$. Note that if $\mu$ is simply chosen to be 0 , then we have a simpler bound depending on $\operatorname{sep}\left(A, T_{n}\right)$; but this gap is usually small because of convergence of the eigenvalues of $T_{n}$ to those of $A$.

We further consider a simplified special case where $r_{i}$ becomes nearly linearly dependent because of the appearance of spurious Ritz values and Ritz vectors (in the underlying Lanczos process). It is known, in the symmetric case, that gradual appearance of spurious Ritz vectors leads to linear dependence [19, 20]. Our next result will be formulated in terms of the Ritz basis, rather than in terms of the basis $Z_{n}$.

An eigenvalue $\theta$ of $T_{n}$ is called a Ritz value and $Z_{n} u$ is called a corresponding Ritz vector if $T_{n} u=\theta u$. By considering the basis of Ritz vectors rather than $Z_{n}$, the pattern of loss of linear independence becomes clear, i.e., the Ritz vectors corresponding to the spurious Ritz values become nearly linearly dependent on the others. For this reason, we divide Ritz vectors into two groups, one consisting of $n-l$ Ritz vectors $\left(Z_{n} U_{1}\right.$ in the theorem below) that are well linearly independent, and the other consisting of the rest, which are nearly linearly dependent on the first group.

Theorem 3.7. Assume equation (11) and that $z_{1}, \cdots, z_{n}$ are linearly independent. Let $T_{n}=U S U^{*}$ be a Schur decomposition ordered such that the columns of $\left[Z_{n} U_{1}, z_{n+1}\right]$ are linearly independent, where

$$
S=\left(\begin{array}{cc}
n-l & l \\
S_{11} & S_{12} \\
0 & S_{22}
\end{array}\right){ }_{l}^{n-l}, \quad U=\left(\begin{array}{cc}
n-l & l \\
l & U_{1}, U_{2}
\end{array}\right) .
$$

Let $V_{0}^{T} \in \mathbf{R}^{(n-l) \times N}$ be the first $n-l$ rows of $\left[Z_{n} U_{1}, z_{n+1}\right]^{+}$. Then

$$
\left\|r_{n+1}\right\| \leq\left(1+K_{n}\right) \min _{p \in \mathcal{P}_{n-l}, p(0)=1}\left\|p\left(A+\delta A_{n}\right) \hat{r}_{1}\right\|,
$$

where $\hat{r}_{1}=\left(I-\left(A+\delta A_{n}\right) / \theta_{1}\right) \cdots\left(I-\left(A+\delta A_{n}\right) / \theta_{l}\right) r_{1}, \lambda\left(S_{22}\right)=\left\{\theta_{1}, \ldots, \theta_{l}\right\}$,

$$
K_{n}=\left\|\left(A Z_{n}-\mathbf{u} \Delta_{n}\right) U_{1} S_{11}^{-1} V_{0}^{T}\right\| \leq\left(\sqrt{n}\|A\|+\mathbf{u}\left\|\Delta_{n}\right\|\right)\left\|T_{n}^{-1}\right\| \cdot\left\|V_{0}\right\|
$$

and $\delta A_{n}=-\mathbf{u} \Delta_{n} Z_{n}^{+}$.

Proof. Let $q(x)=\left(1-x / \theta_{1}\right) \cdots\left(1-x / \theta_{l}\right)$. Then for any polynomial $p \in \mathcal{P}_{n-l}$ with $p(0)=1, \hat{p}(x)=p(x) q(x)$ is of degree $n$ and $\hat{p}(0)=1$. Then $\hat{p}\left(S_{22}\right)=0$, and thus

$$
\hat{p}(S)=\left(\begin{array}{cc}
\hat{p}\left(S_{11}\right) & M \\
0 & \hat{p}\left(S_{22}\right)
\end{array}\right){ }_{l}^{n-l}=\left(\begin{array}{cc}
\hat{p}\left(S_{11}\right) & M \\
0 & 0
\end{array}\right)_{l}^{n-l},
$$

where $M$ is some matrix. Now, as in the proof of Theorem 3.5, we rewrite (11) as

$$
\left(A+\delta A_{n}\right) Z_{n}=Z_{n} T_{n}-\frac{1}{\alpha_{n}^{\prime}} \frac{r_{n+1}}{\left\|r_{1}\right\|} e_{n}^{T} .
$$

Applying Lemma 3.2 (12) to the above with $\hat{p}$, we obtain

$$
\begin{aligned}
\hat{p}\left(A+\delta A_{n}\right) z_{1} & =Z_{n} \hat{p}\left(T_{n}\right) e_{1}+c r_{n+1} \\
& =\left[Z_{n} U_{1}, Z_{n} U_{2}\right] \hat{p}(S) U^{*} e_{1}+c r_{n+1} \\
& =\left[Z_{n} U_{1}, 0\right] \hat{p}(S) U^{*} e_{1}+c r_{n+1} .
\end{aligned}
$$


Multiplying the above by $V_{0}^{T}$, we obtain

$$
V_{0}^{T} \hat{p}\left(A+\delta A_{n}\right) z_{1}=\left[I_{n-l}, 0\right] \hat{p}(S) U^{*} e_{1},
$$

where $V_{0}^{T} Z_{n} U_{1}=I_{n-l}$ and $V_{0}^{T} r_{n+1}=0$ by Lemma 3.4. Also, noting that $S^{-1}$ is block upper triangular of the same structure as $S$, we have

$$
\begin{aligned}
\left(A+\delta A_{n}\right) Z_{n} T_{n}^{-1} \hat{p}\left(T_{n}\right) e_{1} & =\left(A+\delta A_{n}\right)\left[Z_{n} U_{1}, Z_{n} U_{2}\right] S^{-1} \hat{p}(S) U^{*} e_{1} \\
& =\left(A+\delta A_{n}\right)\left[Z_{n} U_{1}, Z_{n} U_{2}\right] \operatorname{diag}\left[S_{11}^{-1}, 0\right] \hat{p}(S) U^{*} e_{1} \\
& =\left(A+\delta A_{n}\right)\left[Z_{n} U_{1} S_{11}^{-1}, 0\right] \hat{p}(S) U^{*} e_{1} \\
& =\left(A+\delta A_{n}\right) Z_{n} U_{1} S_{11}^{-1}\left[I_{n-l}, 0\right] \hat{p}(S) U^{*} e_{1} \\
& =\left(A Z_{n}-\mathbf{u} \Delta_{n}\right) U_{1} S_{11}^{-1} V_{0}^{T} \hat{p}\left(A+\delta A_{n}\right) z_{1} .
\end{aligned}
$$

Now, by (17), we have

$$
\begin{aligned}
\frac{r_{n+1}}{\left\|r_{1}\right\|} & =\hat{p}\left(A+\delta A_{n}\right) z_{1}-\left(A+\delta A_{n}\right) Z_{n} T_{n}^{-1} \hat{p}\left(T_{n}\right) e_{1} \\
& =\left(I-\left(A Z_{n}-\mathbf{u} \Delta_{n}\right) U_{1} S_{11}^{-1} V_{0}\right) \hat{p}\left(A+\delta A_{n}\right) z_{1}
\end{aligned}
$$

Hence

$$
\left\|r_{n+1}\right\| \leq\left(1+K_{n}\right)\left\|\hat{p}\left(A+\delta A_{n}\right) z_{1}\right\|\left\|r_{1}\right\|=\left(1+K_{n}\right)\left\|p\left(A+\delta A_{n}\right) \hat{r}_{1}\right\| .
$$

which leads to the theorem by taking $p$ to be the minimizing polynomial.

Essentially, the above bound replaces $Z_{n}$ (containing $n$ vectors) in Theorem [3.5. which may become nearly linearly dependent, by $Z_{n} U_{1}$ (containing $n-l$ vectors). This eases the dependence of the bound on loss of linear independence among the $z_{i}$. In doing so, however, the degree of the minimizing polynomial is reduced to $n-l$ and thus the convergence is expected to slow down.

\section{NumERICAL EXPERIMENTS}

We present some numerical examples in this section. The purposes of these experiments are to verify the bounds, and to observe how the different parameters in the bounds affect the convergence behavior. All quantities in the bounds are computed with quadruple precision.

We examine the following residual bound (Theorem 3.5) in our numerical experiment:

$$
\left\|r_{n+1}\right\| \leq\left(1+K_{n}\right) \epsilon_{n}
$$

where $\epsilon_{n}=\min _{p \in \mathcal{P}_{n}, p(0)=1}\left\|p\left(A+\delta A_{n}\right) r_{1}\right\|$ is the residual norm of GMRES applied to $A+\delta A_{n}, K_{n}=\left\|\left(A Z_{n}-\mathbf{u} \Delta_{n}\right) T_{n}^{-1} V_{0}^{T}\right\|$ and $\delta A_{n}=-\mathbf{u} \Delta_{n} Z_{n}^{+}$.

Example 1. Our first example is a diagonal matrix $A(N=32)$ with $A(i, i)=$ $1.01^{i}$. We consider injecting respectively $1 \%$ and $10 \%$ random perturbations in the computation of $r_{n+1}$ (but not in $\tilde{r}_{n+1}$ ) and compare the residual norms with the unperturbed case. Note that most known properties of BiCG except the perturbed equation (11) have been lost under the perturbations of this magnitude. In Figure 1 we give the convergence curve for the perturbed (solid) and the unperturbed (dashed) cases and the bound (the + sign) for the perturbed case. To further analyze the various quantities in the bound, we give the magnitudes of $K_{n}$ and the minimum polynomials $\epsilon_{n}$ in Figure 2 and the values $\left\|T_{n}^{-1}\right\|$ and $\left\|V_{0}\right\|$, which bound $K_{n}$, in Figure 3 

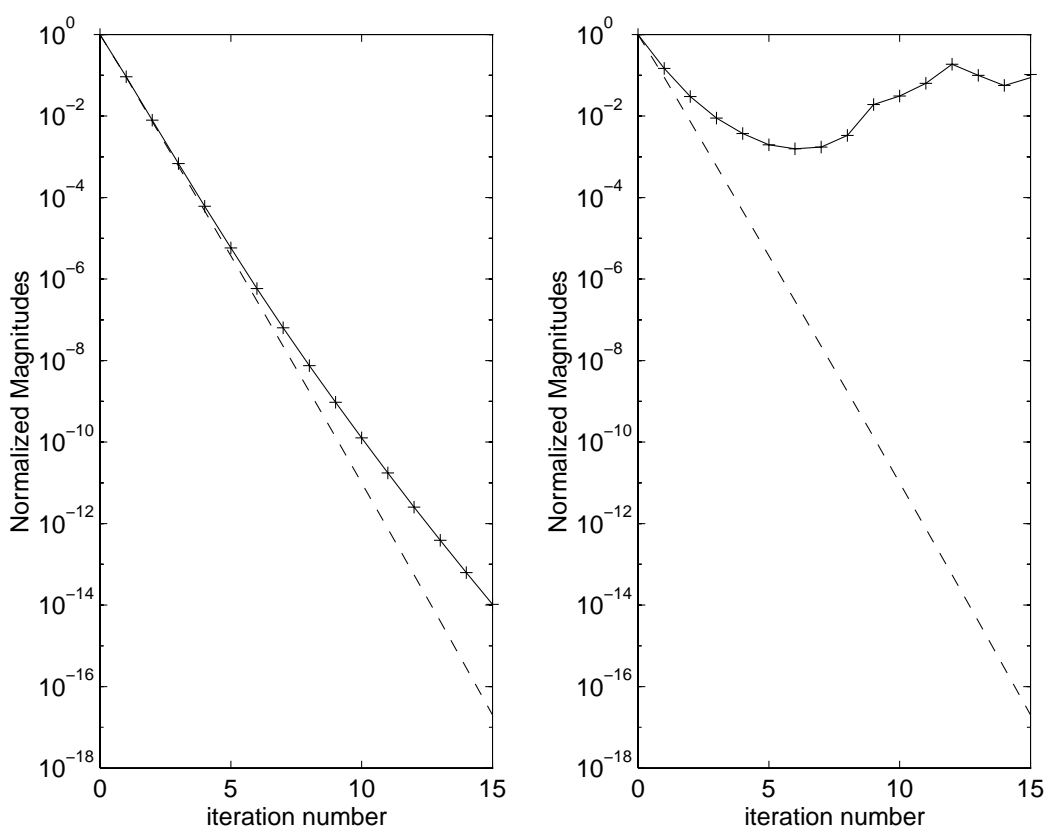

Figure 1. Perturbed BiCG convergence history and bounds in Example 1; (left) 0.01, (right) 0.1 perturbation (solid - with perturbation, dashed - no perturbation, '+' - residual bound)
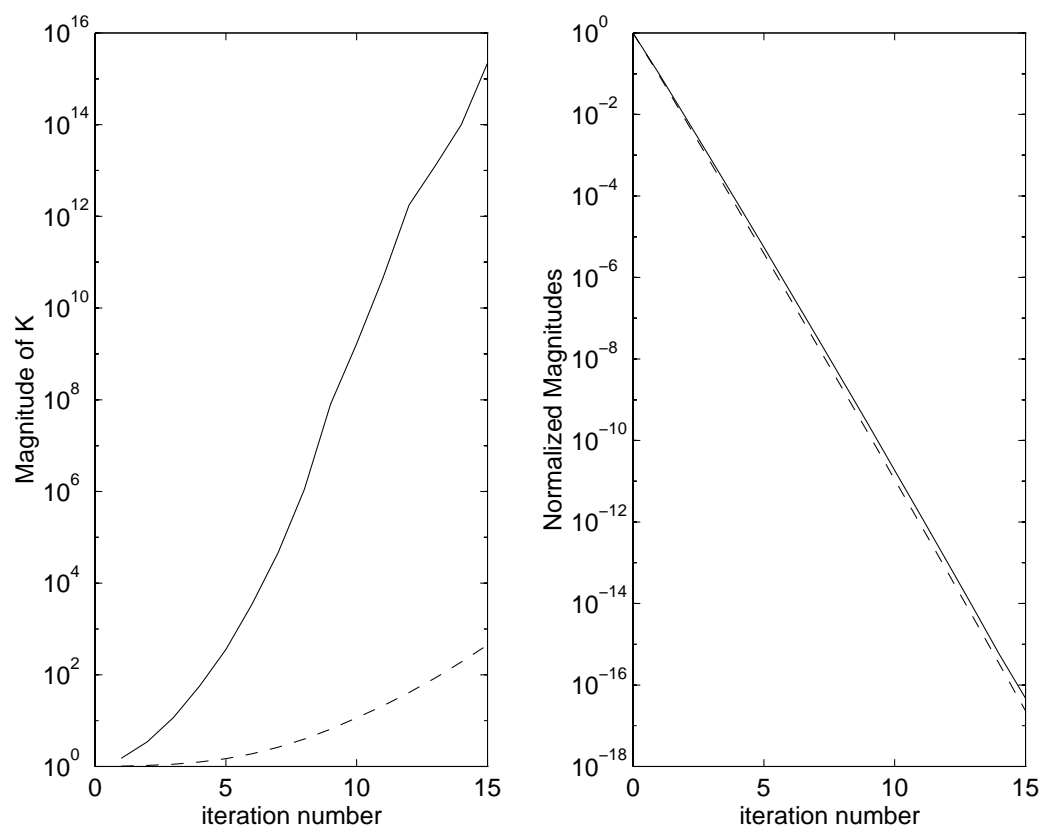

Figure 2. BiCG bounds in Exampleप: (left) values of $K_{n}$, (right) GMRES residual norm $\epsilon_{n}$ (solid - 0.1, dashed - 0.01 perturbation) 

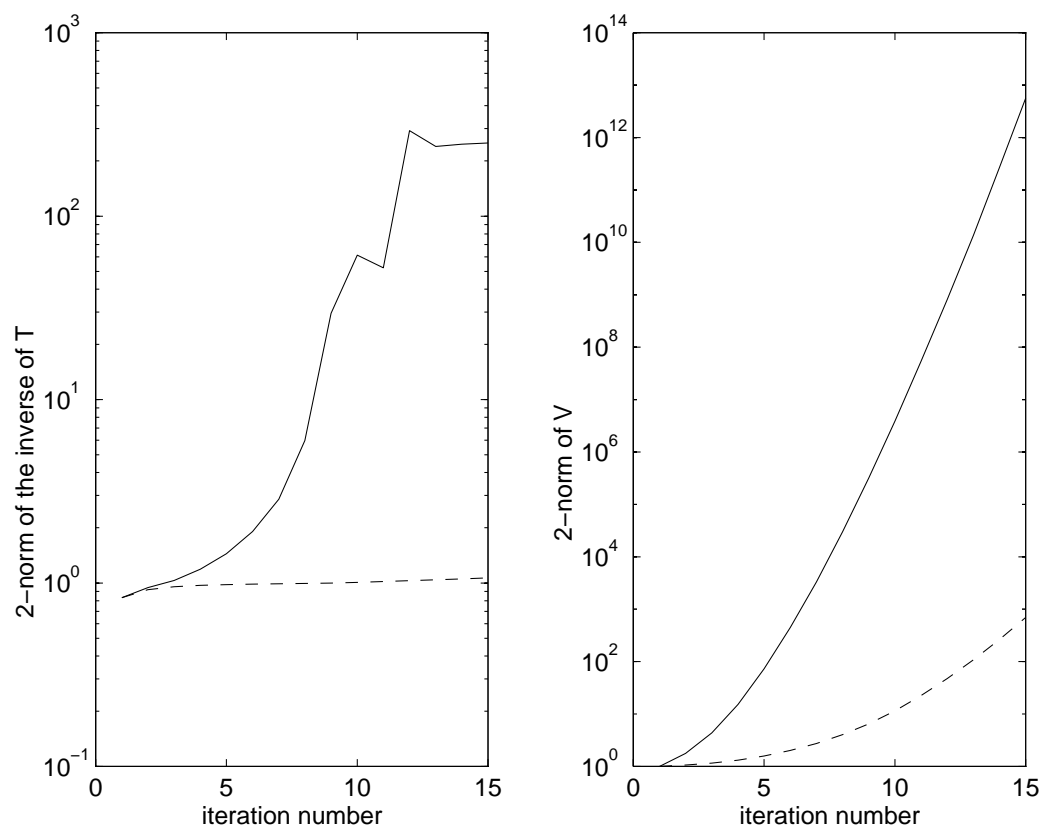

Figure 3. Components of $K_{n}$ in Example 1 (left) $\left\|T_{n}^{-1}\right\|$ (right) $\left\|V_{0}\right\|$ (solid - 0.1, dashed - 0.01 perturbation)
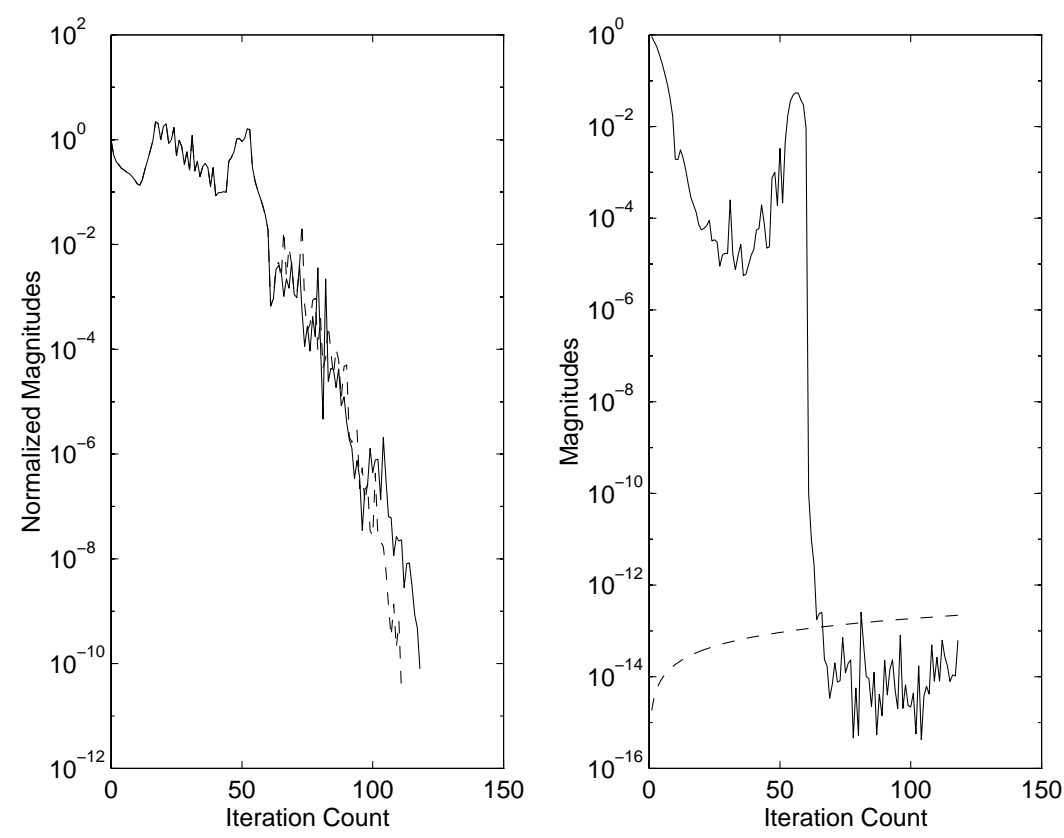

Figure 4. BiCG for the convection diffusion equation in Example 2 (left) convergence history (solid - double precision, dashed quadruple precision), (right) Lanczos pivots $\cos \left\langle r_{n}, \tilde{r}_{n}\right\rangle$ (solid) and roundoff error term $\mathbf{u}\left\|\Delta_{n}\right\|_{F}$ (dashed) 
We first observe that the given bound is satisfied (see Figure 1). We also observe that with $1 \%$ injected error, the BiCG residual still converges to the desired precision, while it diverges for $10 \%$ injected error. Since $\epsilon_{n}$ converges monotonically to 0 at essentially the same rate for both cases, the reason for the divergence in the latter case is that the $K_{n}$ term grows faster than the GMRES contraction $\epsilon_{n}$. A further study on $K_{n}$ (Fig. 3) reveals that $\left\|V_{0}\right\|$, which is a measure of loss of linear independence among the $r_{i}$, plays a major role in determining convergence or divergence. Therefore, it appears that with the smaller injected error (1\%) and relatively short iterations, the loss of linear independence is modest and $\left\|V_{0}\right\|$ grows slowly, so that the GMRES reduction $\epsilon_{n}$ can still drive the residual to convergence. However, with the larger injected error (10\%), the loss of linear independence is much more severe and fast $\left(\left\|V_{0}\right\|\right.$ increases to $\left.10^{12}\right)$, which results in divergence.

In a separate experiment, we inject artificial perturbations in the computation of $\alpha_{i}$ instead of $r_{i}$. Similar behavior was observed. We omit the details.

Example 2. The second numerical example investigates the effect of near-breakdowns on the convergence of BiCG. The matrix used here is the following convection diffusion equation discretized on a $31 \times 31$ uniform rectangular grid:

$$
-\triangle u+50\left(x u_{x}+y u_{y}\right)-25 u=f(x, y) \quad \text { on } \quad \Omega=[0,1]^{2} .
$$

Near-breakdowns occur in this example, and we run BiCG in double and quadruple precisions to show the impact of the roundoff errors. Figure 4 (left) shows the convergence curves of BiCG in double and quadruple precisions, and Figure 4 (right) shows the magnitude of the Lanczos pivots

$$
\cos \left\langle r_{n}, \tilde{r}_{n}\right\rangle=\tilde{r}_{n}^{T} r_{n} /\left(\left\|\tilde{r}_{n}\right\|_{2}\left\|r_{n}\right\|_{2}\right)
$$

and the rounding error term $\mathbf{u}\left\|\Delta_{n}\right\|_{F}$ for the double precision case.

We observe that the two convergence curves start to deviate at around iteration 60, after a near-breakdown occurs (the Lanczos pivot drops to about $10^{-14}$; see Fig. 4). Indeed, the residual vectors as generated by the double and quadruple precisions are completely different after this point. Despite this drop in the Lanczos pivot, however, $\beta_{n}$ remains small throughout, and so is the Frobenius norm of the error term $\mathbf{u} \Delta_{n}$. This confirms the analysis of Theorem 3.1, namely, occurrence of near breakdown does not necessarily cause large error $\mathbf{u} \Delta_{n}$ in the fundamental equation (11). Thus, it does not come as a surprise that the iteration counts required to achieve convergence are very close for the double and quadruple precision cases. In other words, the residual vectors in double and quadruple precision may be completely different, but their norms, bounded by similar quantities, may still be comparable.

\section{Conclusion}

The analysis presented in this paper attempts to shed some light on the important computational issue of how roundoff errors affect convergence of the BiCG algorithm. In particular, we show that even in the presence of roundoff errors, the BiCG residual bound can still be formulated in a way similar to the exact case in terms of a GMRES residual norm. There is, however, an amplification factor $K_{n}$, which cannot be bounded analytically without any a posteriori information. We remark that this is also the case even in exact arithmetic (see [2]), and it seems to be the nature of BiCG type algorithms that convergence cannot be determined $a$ 
priori. The BiCG residual bound given here also suggests and explains that it is still possible to achieve convergence even with relatively large errors in the recurrences, as confirmed by some numerical results.

\section{ACKNOWLEDGMENTS}

We are grateful to Professors Tony Chan, Gene H. Golub, Anne Greenbaum and Beresford Parlett and Dr. David Day for discussions and comments. In particular, we thank Gene Golub for pointing out a connection to inexact CG, and Anne Greenbaum for showing us her related work [15].

\section{REFERENCES}

1. Z. Bai, Error Analysis of the Lanczos Algorithm for the Nonsymmetric Eigenvalue Problem, Math. Comp., 62:209-226 (1994). MR 94c:65045

2. R. E. Bank and T. F. Chan, An Analysis of the Composite Step Biconjugate Gradient Algorithm for Solving nonsymmetric Systems, Numer. Math., 66:295-319 (1993). [MR 94i:65043

3. T. Barth and T. A. Manteuffel, Variable Metric Conjugate Gradient Methods, Proceedings of the 10th International Symposium on Matrix Analysis and Parallel Computing, Keio University, Yokohama, Japan, March 14-16, 1994.

4. J. Cullum and A. Greenbaum, Relation between Galerkian and norm-minimizing iterative methods for solving linear systems SIAM J. Matrix Anal. Appl. 17:223-247 (1996). MR 97b:65035

5. D. Day, Semi-duality in the two-sided Lanczos algorithm. Ph.D. Thesis, University of Californial, Berkeley, 1993.

6. I. S. Duff, R. G. Grimes, and J. G. Lewis, Sparse Matrix Test Problems, ACM Trans. Math. Softw., 15:1-14 (1989).

7. R. Fletcher, Conjugate Gradient Methods for Indefinite Systems, in Proc. Dundee Conference on Numerical Analysis, 1975, Lecture Notes in Mathematics 506, G. A. Watson, ed., SpringerVerlag, Berlin, pp. 73-89 (1976). MR 57:1841

8. R. W. Freund and N. M. Nachtigal, QMR : a Quasi-minimal Residual Method for nonHermitian Linear Systems, Numer. Math., 60:315-339 (1991). MR 92g:65034

9. G. H. Golub and M. L. Overton, Convergence of a two-stage Richardson iterative procedure for solving systems of linear equations. in Numerical Analysis, Lect. Notes Math 912, (ed. G.A. Watson), Springer, New York Heidelberg Berlin pp.128-139. MR 83f:65045

10. G. H. Golub and M. L. Overton, The convergence of inexact Chebyshev and Richardson iterative methods for solving linear systems. Numer. Math. 53:571-593 (1988). MR 90b:65054

11. G. H. Golub, C. F. Van Loan, Matrix Computations, The Johns Hopkins University Press, Baltimore, 1983. MR 85h:65063

12. A. Greenbaum, Behavior of Slightly Perturbed Lanczos and Conjugate-Gradient Recurrences, Lin. Alg. and its Appl., 113:7-63 (1989). MR 90e:65044

13. A. Greenbaum, Accuracy of Computed Solutions from Conjugate-Gradient-Like Methods, PCG '94, Matrix Analysis and Parallel Computing, Keio University, March 14-16, 1994.

14. A. Greenbaum and Z. Strakos, Predicting the behavior of finite precision Lanczos and Conjugate Gradient computations, SIAM J. Matrix Anal. Appl. 13:121-137 (1992). MR 92j:65043

15. A. Greenbaum, V. Druskin, and L. Knizhnerman, Private communication.

16. C. Lanczos, Solution of Systems of Linear Equations by Minimized Iterations, J. Res. Natl. Bur. Stand. 49:33-53 (1952). MR 14:501g

17. R. Lehoucq, Analysis and implementation of an implicitly restarted iteration Ph.D. Thesis, Rice University, Houston, Texas, May 1995.

18. Y. Notay, On the convergence rate of the conjugate gradients in presence of rounding errors, Numer. Math. 65:301-317 (1993). MR 94j:65050

19. C. Paige, Error Analysis of the Lanczos Algorithm for Tridiagonalizing a Symmetric Matrix, J. Inst. Math. Appl., 18:341-349 (1976). MR 58:19082

20. C. Paige, Accuracy and Effectiveness of the Lanczos Algorithm for the Symmetric Eigenproblem, Linear Alg. Appl. 34(1980):235-258. MR 82b:65025

21. Y. Saad, Analysis of some Krylov subspace approximations to the matrix exponential operators, SIAM J. Numer. Anal. 29:209-228 (1992). MR 92m:65050 
22. P. Sonneveld, CGS, a Fast Lanczos-type Solver for Nonsymmetric Linear Systems, SIAM J. Sci. Stat. Comput., 10:36-52 (1989). MR 89k:65052

23. C. H. Tong, A Comparative Study of Preconditioned Lanczos Methods for Nonsymmetric Linear Systems, Tech. Report SAND91-9240B, Sandia National Lab., Livermore, 1992.

24. L. N. Trefethen, Approximation theory and numerical linear algebra, in Algorithms for Approximation II, J.C. Mason and M.G. Cox eds., Chapman and Hall, London, 1990, pp. 336360. MR 91j:65063

25. H. A. Van der Vorst, Bi-CGSTAB : A Fast and Smoothly Converging Variant of Bi-CG for the Solution of Nonsymmetric Linear Systems, SIAM J. Sci. Stat. Comput., 13:631-644 (1992). MR 92j:65048

26. H. A. Van der Vorst, The convergence behaviour of preconditioned $C G$ and $C G S$ in Lect. Notes in Math. 1457, ed. O. Axelsson and L. Kolotilina, pp. 121-136, Springer, Berlin Heidelberg New York (1990), pp. 126-136. MR 92a:65141

27. Q. Ye, A convergence analysis of nonsymmetric Lanczos algorithms, Math. Comp. 56:677-691 (1991). MR 91m:65115

Sandia National Laboratories, Livermore, CA 94551

E-mail address: chtong@california.sandia.gov

Department of Mathematics, University of Manitoba, Winnipeg, Manitoba, Canada R3T $2 \mathrm{~N} 2$

E-mail address: ye@gauss.amath.umanitoba.ca 